\title{
Modeling and Applications of Electrochemical Impedance Spectroscopy (EIS) for Lithium-ion Batteries
}

\author{
Woosung Choi ${ }^{1}$, Heon-Cheol Shin ${ }^{2}$, Ji Man Kim ${ }^{3}$, Jae-Young Choi ${ }^{4,5}$, and Won-Sub Yoon ${ }^{1 *}$ \\ ${ }^{1}$ Department of Energy Science, Sungkyunkwan University, Suwon 440-746, South Korea \\ ${ }^{2}$ School of Materials Science and Engineering, Pusan National University, Busan 46241, Republic of Korea \\ ${ }^{3}$ Department of Chemistry, Sungkyunkwan University, Suwon, 16419, South Korea \\ ${ }^{4}$ School of Advanced Materials Science and Engineering, Sungkyunkwan University (SKKU), Suwon, Gyeonggi 16419, \\ Republic of Korea \\ ${ }^{5}$ SKKU Advanced Institute of Nanotechnology (SAINT), Sungkyunkwan University (SKKU), Suwon, Gyeonggi 16419, \\ Republic of Korea
}

\begin{abstract}
As research on secondary batteries becomes important, interest in analytical methods to examine the condition of secondary batteries is also increasing. Among these methods, the electrochemical impedance spectroscopy (EIS) method is one of the most attractive diagnostic techniques due to its convenience, quickness, accuracy, and low cost. However, since the obtained spectra are complicated signals representing several impedance elements, it is necessary to understand the whole electrochemical environment for a meaningful analysis. Based on the understanding of the whole system, the circuit elements constituting the cell can be obtained through construction of a physically sound circuit model. Therefore, this mini-review will explain how to construct a physically sound circuit model according to the characteristics of the battery cell system and then introduce the relationship between the obtained resistances of the bulk $\left(\mathrm{R}_{\mathrm{b}}\right)$, charge transfer reaction $\left(\mathrm{R}_{\mathrm{ct}}\right)$, interface layer $\left(\mathrm{R}_{\mathrm{SEI}}\right)$, diffusion process $(\mathrm{W})$ and battery characteristics, such as the state of charge (SOC), temperature, and state of health ( $\mathrm{SOH})$.
\end{abstract}

Keywords : Electrochemical Impedance Spectroscopy, Lithium-ion Batteries, Equivalent Circuit Model, Diagnosis of Battery Characteristics

Received : 4 September 2019, Accepted : 27 October 2019

\section{Introduction}

There are many advantages to using electrochemical impedance spectroscopy (EIS) for understanding the power delivery capability in lithium-ion battery systems. EIS can separate and quantify the cell resistance of the bulk $\left(\mathrm{R}_{\mathrm{b}}\right)$, interface layer $\left(\mathrm{R}_{\mathrm{SEI}}\right)$, charge transfer reaction $\left(\mathrm{R}_{\mathrm{ct}}\right)$, and diffusion process $(\mathrm{W})$ by a single experiment. Measuring EIS spectra does not require disassembly of the battery cell, which is very advantageous in preventing sensitive samples from being contaminated with moisture and oxygen. It is also possible to carry out EIS measurements under

*E-mail address: wsyoon@skku.edu

DOI: https://doi.org/10.33961/jecst.2019.00528

This is an open-access article distributed under the terms of the Creative Commons Attribution Non-Commercial License (http://creativecommons.org/licenses/by-nc/4.0) which permits unrestricted non-commercial use, distribution, and reproduction in any medium, provided the original work is properly cited. operating conditions, which is helpful for obtaining spectra without any disrupting influences on the cell. Finally, EIS analyses need only a small amount of time and cost.

This powerful analysis tool was firstly initiated by Oliver Heaviside, proposing the concept of "impedance" in the 19th century [1]. Subsequently, Warburg was able to include lithium-ion transportation in the impedance spectra interpretation by defining the term "Warburg impedance", which is associated with the chemical diffusion process [2]. Since then, the development of bridge models has been spurred through the study of diverse combinations of circuit elements [3]. In the 1950s, the capacitive characteristics of the electrical double layer deposited on the electrode surface were outlined by D.C. Grahame [4]. Along with the advancement of theories and measurement methods, the development of an EIS analyzer called a dynamic "frequency response analyzer" (FRA) 
enabled the measurement of impedance spectra at frequencies less than $1 \mathrm{mHz}$ in the late 1970s [5-8]. With this continued technological improvement, research on EIS has been carried out in various fields such as corrosion [9-11], catalyst [12,13], supercapacitor [14,15], and fuel cell [16-18]. However, handling of EIS spectra is not very straight forward. To analyze complex electrochemical systems, such as lithium-ion batteries, it is essential to understand the whole system first. By understanding the system completely, a physically sound circuit model can be constructed, and this model is necessary to interpret the characteristics of the impedance spectrum.

Basically, a lithium-ion battery is composed of a cathode, an anode, a separator, current collectors, and an electrolyte. During electrochemical reactions, electrons and lithium ions pass through each component of the battery that has resistive and capacitive properties. In other words, the battery components and the double layer on the interface are the circuit elements constituting the lithium-ion battery circcuit model, which connects these components in parallel and/or series. Although the impedance properties of the circuit elements vary depending on the cell type and character of the components [19], generally the total impedance value can be obtained by scanning the $\mathrm{AC}$ frequency in the $100 \mathrm{kHz} \sim 10 \mathrm{mHz}$ range $[20,21]$. The frequency range of EIS analysis is depending on typical response time with contained cell's components. Therefore, the slow transport process of chemical lithium diffusion which denoted as Warburg impedance represented in the low-frequency of $10 \mathrm{~Hz}$ to $10 \mathrm{mHz}$. And subsequently followed by the typical times constants, the impedance related to the charge transfer reaction can be obtained in the middle-frequency range of $10 \mathrm{kHz}$ to $10 \mathrm{~Hz}$ region and the relatively fast transport process through the interface layer is measured in the highfrequency of $100 \mathrm{kHz}$ to $10 \mathrm{kHz}$. The corresponding impedance values for each frequency range can be analyzed through EIS spectral fitting based on the determined equivalent circuit model [22].

In this mini-review, the basic principles of EIS and the process of constructing a meaningful equivalent circuit model for lithium-ion batteries are introduced. Then, practical applications of EIS for understanding lithium-ion battery systems are explained.

\section{Principle of EIS}

EIS analysis is a technique that measures resistance $(\mathrm{R})$, capacitance $(\mathrm{C})$, and inductance (L) by monitoring the current response while an AC voltage is applied to an electrochemical cell.

When a DC voltage is applied, the relation among R, V and I satisfy Ohm's law, as shown in Fig. 1(a). Similarly, when an AC voltage is applied to an electrochemical cell, the impedance $Z(\omega)(\omega=2 \pi f$ is the angular frequency of the applied AC voltage) can be expressed as $\mathrm{V}(\omega) / \mathrm{I}(\omega)$, which is the equation for Ohm's law in an AC circuit. Therefore, the impedance is defined as the resistance that interrupts the current flow when an AC voltage is applied to the circuit. These interruptions are represented by various circuit elements, such as resistors, inductors, and conductors, which constitute the overall impedance in a circuit. In an actual system, the electrochemical cell contains various circuit elements with diverse combinations. Thus, it is more accurate to use the concept of impedance than a circuit model consisting solely of a resistor to represent an electrochemical system. Additionally, when $\mathrm{V}$ is applied with the angular frequency $\omega, \mathrm{V}$ and $\mathrm{I}$ have a phase difference of $\varnothing$. Therefore, the above $\mathrm{V}$ and I values in the AC circuit can be expressed by the equation shown in Fig. 2. In these equations, $\mathrm{V}_{\mathrm{m}}$ and $\mathrm{I}_{\mathrm{m}}$ represent the maximum values of $\mathrm{V}$ and $\mathrm{I}$, respectively. (a) DC voltage

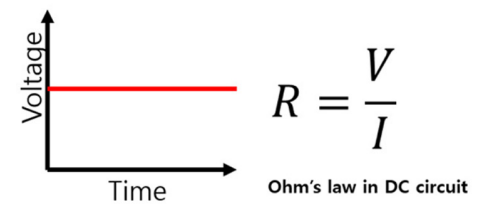

(b) AC voltage

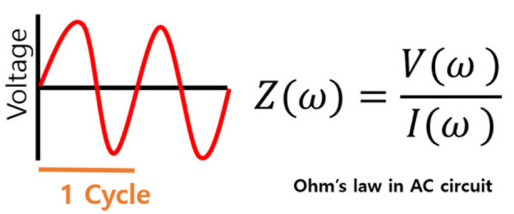

Fig. 1. Schematic diagram and equation showing Ohm's law for (a) DC circuits where DC voltages are formed by direct current and (b) $\mathrm{AC}$ circuits from which $\mathrm{AC}$ voltage is derived by alternating current. 


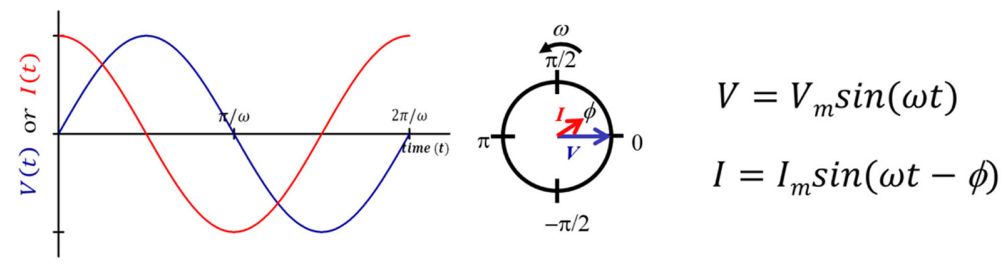

Fig. 2. The illustration and equation about the relationship between the voltage and current when applying an AC voltage with the angular frequency $\omega$.

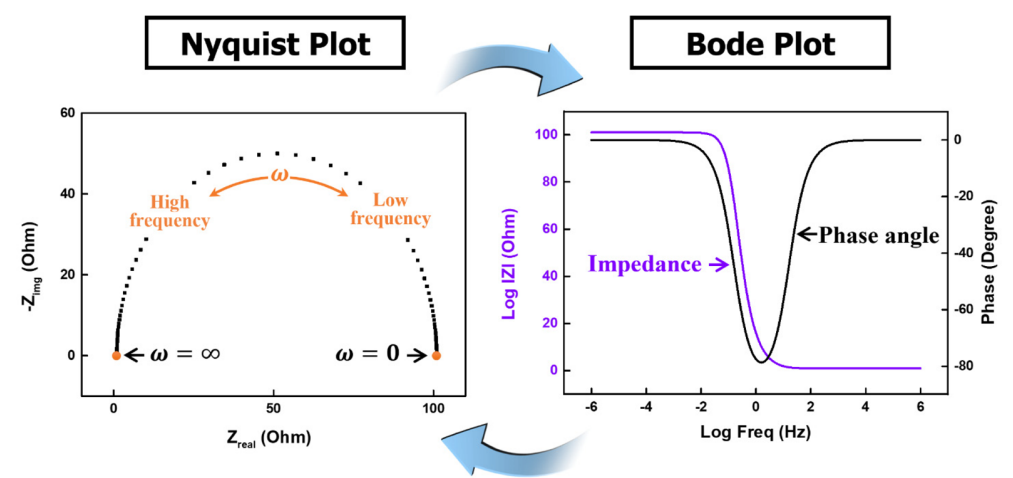

Fig. 3. Electrochemical Impedance Spectroscopy representation by Nyquist plot (left) and Bode plot (right).

The above equation for $\mathrm{V}$ and I can be moditied by using the complex function $j=\sqrt{-1}=\exp \left(\frac{j \pi}{2}\right)$.

$$
\begin{aligned}
& V=V_{m} \exp (j \omega t) \\
& I=I_{m} \exp [j(\omega t-\varnothing)]
\end{aligned}
$$

V and I satisfy Ohm's law for the AC circuit model shown in Fig. 1. Therefore, the impedance $Z(\omega)$ can be expressed as:

$Z(\omega)=\frac{V(\omega)}{I(\omega)}=\frac{V_{m} \exp (j \omega j)}{I_{m} \exp [j(\omega t-\emptyset)]}=\frac{V_{m}}{I_{m}} \exp (j \emptyset)$

Equation (3) can be simplified using Euler's formula, $\exp (j \varnothing)=\cos (\varnothing)+j \sin (\varnothing)$. Thus, equation (3) becomes:

$$
\begin{aligned}
Z(\omega) & =\frac{V_{m}}{I_{m}} \exp (j \varnothing)=\frac{V_{m}}{I_{m}}[\cos (\varnothing)+j \sin (\varnothing)] \\
& =Z_{0}[\cos (\varnothing)+j \sin (\varnothing)]
\end{aligned}
$$

By dividing the above equation into real and imaginary parts, the following equation can be obtained:

$$
Z(\omega)=Z_{0} \cos (\varnothing)+Z_{0} \sin (\varnothing) j
$$

$* Z_{\text {real }}^{\prime}=Z_{0} \cos (\varnothing): \mathrm{R}($ resistance $)$

${ }^{*} Z^{\prime \prime}{ }_{\text {img }}=Z_{0} \sin (\varnothing): \mathrm{C}($ capacitance $)+\mathrm{L}($ inductance $)$

Based on equation (5), the impedance spectrum can be represented in two different ways: (i) the "Bode plot" that shows the phase shift and magnitude changes in the applied frequency ranges and (ii) the "Nyquist plot" that represents the real and imaginary parts of $Z(\omega)$ using cartesian coordinates. The Bode plot has great advantages for observing phase margins in which the system becomes unstable (violent phase or magnitude changes). Therefore, it is useful for the study of sensors, filters, and transistors in electronic devices. The Nyquist plot provides insight into the possible mechanism or governing phenomena in an equivalent circuit model system. Among these two types of representations, the Nyquist plot is more often used to analyze the characteristics of lithium-ion batteries due to its convenience for analyzing the active reaction mechanism.

\section{Circuit Model}

The circuit model for EIS consists of electrical circuit elements such as resistors (R), capacitors (C), 
and inductors (L). The designated circuit model represents the entire system of the electrochemical cell. Therefore, understanding the circuit model is critically important to understand the entire electrochemical system. To construct an accurate circuit model, suitable electrical components that represent the overall system should be utilized. The aim is to construct an optimal circuit model that is physically meaningful and minimizes the number of variables.

In the case of a lithium-ion battery, additional elements such as the constant phase element (CPE) and Warburg impedance (W) are used to supplement nonideal capacitor and lithium diffusion characteristics, respectively. Between the electrode and the electrolyte, there is an electrical double layer that has capacitive characteristics. However, its characteristics are far from those of an ideal capacitor. Surface roughness, leakage capacitance, and nonuniform distribution have been reported to account for such nonideal behavior. This nonideal behavior of the capacitor can be compensated by using the concept of CPE in the modeling of an electrical double layer [23,24]. Warburg impedance was used to describe the impedance created by the lithium diffusion process. Since a real cell behaves similarly to "semi-infinite" Warburg impedance, i.e., diffusion in one dimension that is only bound by the planar electrode side, the impedance shows $45^{\circ}\left(90^{\circ}\right.$ for capacitor) in the low-frequency region [25]. This will be discussed in detail later. The Warburg impedance in a lithium-ion battery has the following relationship $\mathrm{W}=\sigma \omega^{-1 / 2}-j \sigma \omega^{-1 / 2}(\sigma$ : Warburg coefficient), and its details are shown in Table 1.

The equivalent circuit model construction using the fundamental electrochemical elements is explained as follows.

Table 1. Circuit elements used in the equivalent circuit model.

\begin{tabular}{cc}
\hline \hline Equivalent Element & Formula \\
\hline $\mathrm{R}$ (Resistance) & $\mathrm{R}$ \\
$\mathrm{C}$ (Capacitance) & $1 / \mathrm{j} \omega \mathrm{C}$ \\
$\mathrm{L}$ (Inductance) & $\mathrm{j} \omega \mathrm{L}$ \\
$\mathrm{W}$ (Warburg Impedance) & $\sigma \omega^{-1 / 2}-\mathrm{j} \sigma \omega^{-1 / 2}$, \\
& $\sigma($ Warburg coefficient $)$ \\
$\mathrm{Q}_{\mathrm{CPE}}$ (Constant Phase Element) & $1 / \mathrm{Q}(\mathrm{j} \omega)^{\alpha}$ \\
& $(\alpha=1$ for ideal capacitor $)$ \\
\hline
\end{tabular}

\subsection{Equivalent circuit model of parallel R-C}

The equivalent circuit model and Nyquist plot for a parallel R-C circuit are shown in Fig. 4.

The diameter of the semicircle shown along the real part in Fig. 4(b) indicates the resistance of $R_{1}$ in Fig. 4(a). Therefore, the resistance of $R_{1}$ is 100 $\Omega$, which is the end of the semicircle along the real axis. The Nyquist plot was simulated using a capacitance of $100 \mathrm{mF}$. This capacitance value can be determined from the maximum point of the semicircle in the Nyquist plot. At the center of the semicircle, where $Z_{\text {img }}$ is at a minimum, the values of $\omega_{\min }, \mathrm{R}_{1}$ and $\mathrm{C}_{1}$ satisfy the following equation.

$$
\omega_{\text {min }}=\frac{1}{R_{1} C_{1}}
$$

Therefore, by identifying the $\mathrm{R}_{1}$ and $\omega$ values when $\mathrm{Z}_{\mathrm{img}}$ is at a minimum value, the capacitance value can be calculated by the above equation (6).

\subsection{Simplified Randles cell}

The simplified Randles model includes a double layer capacitor with bulk resistance (current collector, solution, etc.) and polarization resistance. The polarization resistor $\left(R_{1}\right)$ is connected in series with the bulk resistor $\left(R_{2}\right)$ and in parallel with the surface deposited double layer capacitor, as shown in Fig. 5(a). This model is fundamental for analyzing an electrochemical system. As real systems tend to be more complicated, additional elements are connected in this simplified Randles cell. The Nyquist plot of a simplified Randles cell is shown in Fig. 5(b).

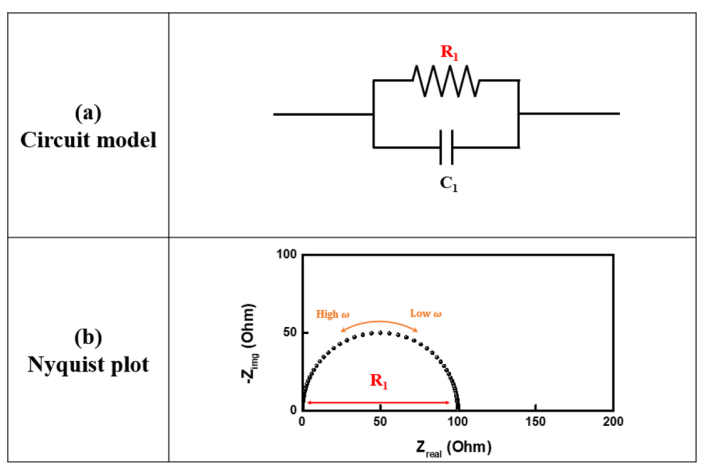

Fig. 4. (a) Equivalent circuit model and (b) Nyquist plot of a parallel R-C circuit. 
In the Nyquist plot of a simplified Randles cell, the resistance of $R_{2}$ shifts the starting point of the semi-

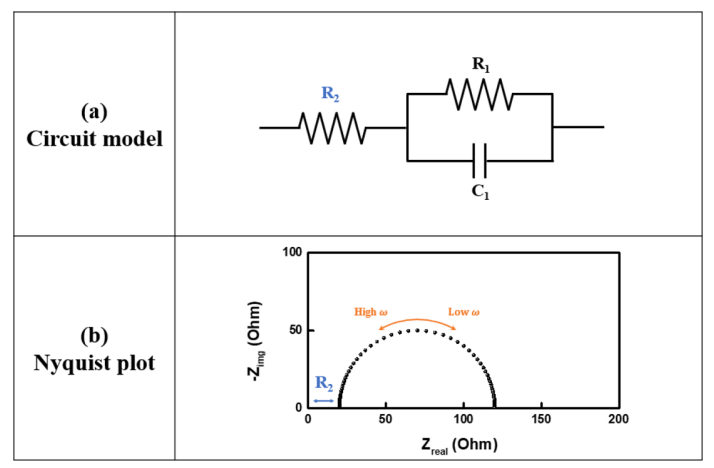

Fig. 5. (a) Equivalent circuit and (b) Nyquist plot of a simplified Randles cell model.

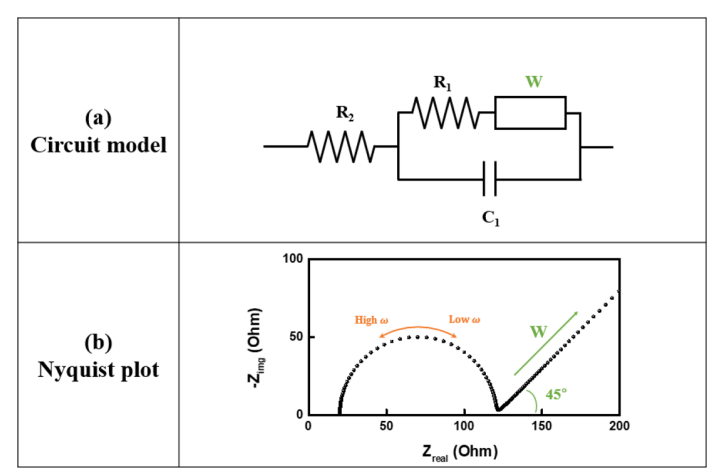

Fig. 6. (a) Equivalent circuit and (b) Nyquist plot of a Randles cell model. circle to higher $Z_{\text {real }}$ values. Considering that this spectrum was generated by $R_{2}=20 \Omega$ and $R_{1}=$ $100 \Omega$, the $120 \Omega$ at the end of this semicircle (lowfrequency) intercept on the $\mathrm{x}$-axis is the sum of the polarization resistance and the bulk resistance. Additionally, the diameter of the semicircle represents the polarization resistance (in this case, $100 \Omega$ ).

\subsection{Mixed kinetic and diffusion control (Randles cell)}

In special cases where semi-infinite linear diffusion affects an electrochemical system by kinetic and diffusion control, the Warburg impedance is used in the equivalent circuit model.

This model was presented by the Faraday Society in 1947, and the name of this model is taken from the name of the creator J.E.B. Randles[26]. The circuit model and the Nyquist plot for this cell are shown in Fig. 6. The Warburg impedance in Fig. 6(b) appears as a straight line with a $45^{\circ}$ slope under semi-infinite conditions, and one-dimensional diffusion that is bound only by a large planar electrode on one side [25]. Along with the bulk and polarization resistance losses, this model represents the polarization in the lithium diffusion reaction as well. The Warburg coefficient, $\sigma$, in this case is $1 \Omega \cdot \mathrm{s}^{-1 / 2}$, and the other values are $\mathrm{R}_{2}=20 \Omega, \mathrm{R}_{1}=100 \Omega$, and $\mathrm{C}_{1}=100 \mathrm{mF}$, which are similar to the previous models.

\subsection{Lithium-ion battery circuit models}

An entire lithium-ion battery cell can be modeled using the equivalent circuit shown in Fig. 7. Although there are still challenges in interpreting the
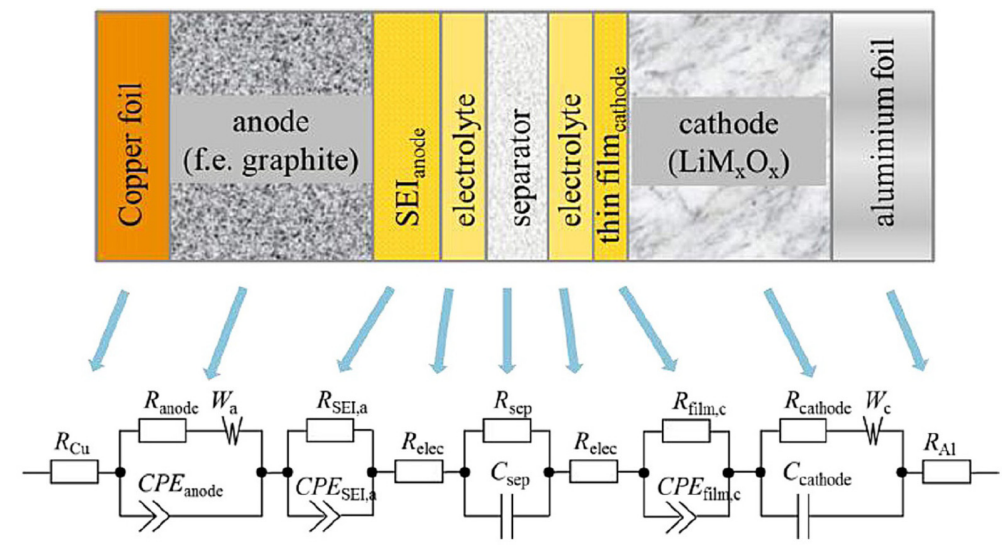

Fig. 7. Entire circuit model of a lithium-ion battery. Reprinted with permission from Ref. [28]. Copyright (C) 2016 Wiley$\mathrm{VCH}$ verlag $\mathrm{GmbH} \& \mathrm{Co}$. KGaA. 
meaning of each semicircle in EIS spectra[27], this model follows the traditional interpretation of each arc. However, since this circuit model has many variables, it is necessary to minimize the circuit elements for practical impedance analysis.

Among these circuit elements, the capacitive effect of the separator is minimal compared to that of the other components. Therefore, it is not necessary to consider $\mathrm{C}_{\text {sep }}$ in setting up a circuit model[28]. Furthermore, the EIS spectra mainly come from the contribution of the working electrode in half-cell conditions, so the model does not need to consider the counter electrode. Additionally, the resistances $\mathrm{R}_{\text {current collector }}, \mathrm{R}_{\text {electrolyte, and }} \mathrm{R}_{\text {separator }}$ can be combined into the bulk resistance $R_{b}$. Moreover, since the capacitances of lithium-ion battery components are not ideal values, it is possible to obtain a more accurate model by replacing them with a constant phase element. The overall simplified equivalent circuit of a lithium-ion battery half-cell is shown in Fig. 8.

However, in the case of a half-cell system, the impedance value coming from the lithium counter electrode may affect the entire impedance spectrum. Therefore, in a certain case where the lithium counter electrode causes a relatively large impedance, there is an advanced measuring method assembling a sym-
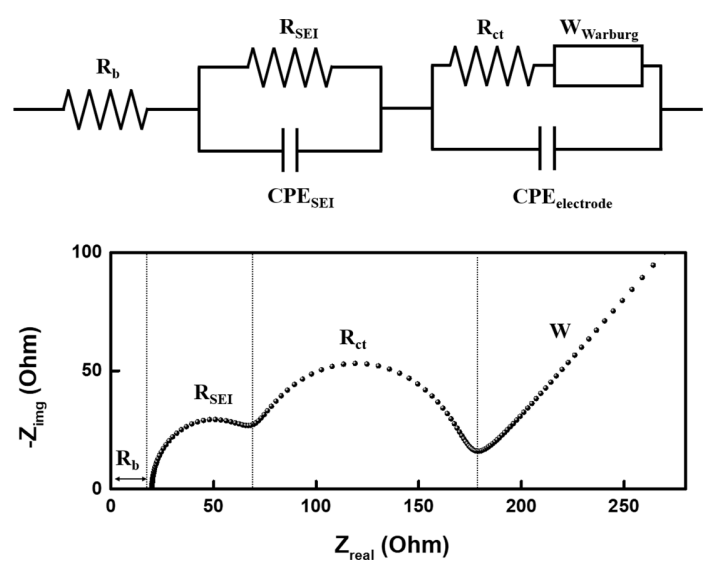

Fig. 8. Equivalent circuit model of a lithium-ion battery half-cell system.

- $\mathbf{R}_{\mathbf{b}}$ : bulk resistance of the cell (electrolyte, separator, anc electrodes)

- $\mathbf{R}_{\mathrm{SEI}}, \mathbf{C P E _ { \mathrm { SEI } }}$ : resistance and capacitance of the interfacial layer

- $\mathbf{R}_{\mathrm{ct}}, \mathbf{C P E}_{\text {electrode: }}$ : charge-transfer resistance and doublelayer capacitance

- W: diffusional effects of lithium-ion on the host material metric cell [29] and a three-electrode cell [27,30] that only get the EIS spectra from the target electrode.

The above circuit model is based on the most fundamental lithium-ion battery half-cell system. It is useful to understand fundamental cell models; however, an equivalent circuit model representing a lithium-ion battery can be diverse depending on the electrode characteristics, cell type, and storage and cycling conditions [31]. Therefore, there is no single standard circuit model that may fit all battery systems. A customized circuit model can be devised by adding or subtracting electronic elements depending on the electrochemical cell characteristics [32-35].

After configuring the circuit model, it is necessary to ensure its accuracy. Zhai et al. reported the EIS determination algorithm using the digital signal processing (DSP) system and PC communication through ethernet/RS232 communication [36]. The EIS determination algorithm repeatedly matches the EIS fitting curve with the experimental data until the unknown value is obtained based on constructed circuit model. An open-source platform, the Impedance Analyzer, for easy-to-use physics-based analysis of experimental EIS spectra is available as well [37].

\section{EIS Application to Lithium-ion Batteries}

\subsection{Characterization of $R_{b}$ in EIS spectra}

$R_{b}$ represents the internal resistance value of the bulk materials in a battery, such as the current collector, electrolyte and separator. Since these values have a resistance only without capacitance or inductance, their effect can be observed in $Z_{\text {real }}$. This bulk resistance does not change significantly with the state of charge, but as cycling continues, the bulk resistance increases due to the depletion of the electrolyte and microcrack formation in the particles. Therefore, the bulk resistance is generally used as an indicator to determine the state of health ( $\mathrm{SOH})$ of aged cells.

Westerhoff et al. monitored the change in the internal resistance of a lithium-ion battery while the state of health decreased from $100 \%$ to $86 \%$, as shown in Fig. 9(a) [28]. During the aging process, the impedance spectra moved gradually to higher $Z_{\text {real }}$ values, and the shape of the semicircle changed as well. This change in the semicircle can be explained based on the frequency defined in Fig. 9(b). The starting point of impedance spectroscopy is referred to as $f_{\mathrm{ZIM}, 0}$ indicating purely ohmic resistance, and the maximum 

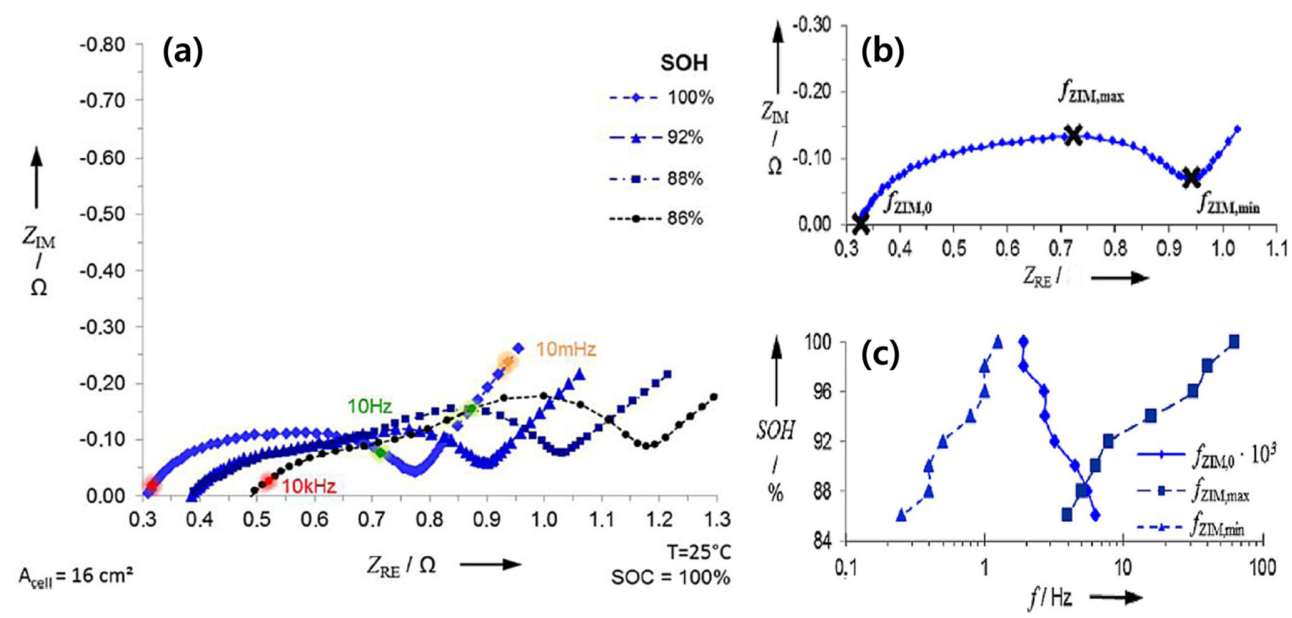

Fig. 9. (a) The impedance spectra change as the state of health decrease from $100 \%$ to $86 \%$. (b) The position of $f_{\text {ZIM }, 0}$, $f_{\mathrm{ZIM}, \max }$ and $f_{\mathrm{ZIM}, \min }$ values in the impedance spectra and (c) the changes of $f_{\mathrm{ZIM}, 0}, f_{\mathrm{ZIM}, \max }$ and $f_{\mathrm{ZIM}, \min }$ values depending on the state of health. Reprinted with permission from Ref. [28]. Copyright (C) 2016 Wiley-VCH verlag GmbH\&Co. KGaA.

of the semicircle in the middle of the impedance spectrum correlates to the charge transfer process and is expressed as $f_{\text {ZIM,max }}$. The starting frequency of the

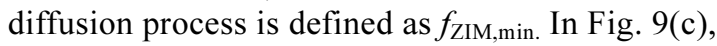
the charge transfer associated with $f_{\text {ZIM,max }}$ and lithium diffusion associated with $f_{\mathrm{ZIM} \text {,min }}$ moved to the lower $f$ values as SOH decreased from $100 \%$ to 86 $\%$. However, the $f_{\text {ZIM }, 0}$ shifts to higher $f$ values, which demonstrates the internal resistance. Therefore, it can be noticed that the total resistance of the aged batteries are coming from the increase in the internal resistance and then the $\mathrm{R}_{\mathrm{b}}$ can be used as an indicator of battery aging during cycling. An increase in the internal resistance was also observed during long-term storage. Eddahech et al. focused on performance degradation with storage time by comparing four different cathode materials (NMC, NCA, LFP, and LMO) with graphite counter electrodes and all of four aged cathodes show increased $R_{b}$ value [38]. In addition, Schuster et al. investigated the aging process using a widespread aging matrix $[39,40]$. These reports suggested the use of $R_{b}$, which is sensitive to operating and storage conditions, as an indicator of the $\mathrm{SOH}$ rather than the use of $\mathrm{R}_{\mathrm{SEI}}$ or $\mathrm{R}_{\mathrm{ct}}$.

The aging of lithium-ion batteries is caused by a combination of diverse phenomena. Formation of microcracks, gas evolution, binder decomposition, corrosion of the current collector and electrolyte depletion have all been reported as the cause of bat- tery aging and performance degradation [41]. It is challenging to distinguish these various processes and identify the characteristics of aging. In many cases, measuring the $\mathrm{R}_{\mathrm{b}}$ using EIS is a very convenient and critical method to evaluate the condition of the aged battery.

\subsection{Characterization of $\mathbf{R}_{\mathrm{SEI}}$ in EIS spectra}

The first semicircle of the EIS spectrum in Fig. 8 originates from the impedance of a layer that forms on the interface between the electrode and electrolyte. This interfacial layer is called the solid electrolyte interphase (SEI) layer, and it is generated by the decomposition of the electrolyte. The SEI layer has a more profound influence on the electrochemical characteristics of the anode material than the cathode material. The characteristics of the layer, such as surface coating, gradient, surface area, etc., are changed. In addition, some nanostructured anode materials, such as $\mathrm{Co}_{3} \mathrm{O}_{4}, \mathrm{Fe}_{2} \mathrm{O}_{3}$, and $\mathrm{MnO}$, shows a reversible film formation that contributes to additional capacity beyond the theoretical capacity [42-46]. Therefore, by monitoring the $\mathrm{R}_{\mathrm{SEI}}$, we can observe the reversible organic film formation as well as identify the characteristics of the SEI layer.

Zhang et al. studied the formation of the SEI layer on the surface of graphite using the EIS technique under various electrochemical cycling conditions [47]. First, the characteristics of the SEI layer were 
studied at different states of charge/discharge. A relatively resistive preliminary SEI is formed at approximately $0.15 \mathrm{~V}$, and then a highly conductive SEI layer forms between 0.15-0.04 V. Accordingly, it can be confirmed that SEI layers with two different characteristics are formed according to the voltage region in the graphite anode. The resistance of the SEI layer also depends on the constituents of diverse electrolytes, such as solvents (EC, EMC, MB, etc.) and lithium salts $\left(\mathrm{LiBF}_{4}, \mathrm{LiSO}_{3} \mathrm{CF}_{3}, \mathrm{LiBOB}, \mathrm{LiPF}_{6}\right.$, etc.). It was found that $\mathrm{R}_{\mathrm{SEI}}$ is very sensitive to the reactivity of the electrolyte during the first lithiation cycle. Furthermore, Steinhauer et al. monitored the SEI formation by calculating the distribution relaxation time (DRT) from the measured impedance spectra to gain more insight into the number of processes involved in the cell [48]. They compared the EIS spectra for 2and 3- electrode cells to study the effect of SEI film formation on the lithium counter electrode. Abarbanel et al. investigated the cause of the impedance increase when $\mathrm{Li}\left[\mathrm{Ni}_{0.42} \mathrm{Mn}_{0.42} \mathrm{Co}_{0.16}\right] \mathrm{O}_{2}(\mathrm{NMC} 442) /$ graphite lithium-ion cells operate at high voltages beyond $4.4 \mathrm{~V}$ using the transmission line model (TLM) [35]. It was found that the impedance increases in the high-frequency region when operat- ing at high voltage are mainly caused by the transfer resistance through the SEI layer along with the diverse influences of the contact resistance (between the carbon conductor and active material), the electrical path resistance through carbon black and the ionic path resistance through the electrolyte solution imbedded in the pore structure.

Moreover, in the impedance spectra of $\mathrm{Li} / \mathrm{C}$ cells, an inductive loop can be observed in some cases because of the unstable nature of the SEI layer. Once a stable SEI has formed, the inductive loop does not appear in the impedance spectra. The formation and disappearance of the inductive loop in the impedance spectra can be used to assess the robustness of the SEI film on the anode [49].

In addition, there is a previous report that reversible SEI formation contributes to extra capacity in high-energy sodium conversion systems. Shubhra et al. observed the impedance spectra of a $\mathrm{MoS}_{2}$ electrode at eight specified potentials, as shown in Fig. 10 [50]. In the initial stage of discharge, $0.8 \mathrm{~V}$, two semicircles are observed, which represent SEI formation and $\mathrm{Na}$ ion intercalated in $\mathrm{MoS}_{2}$ on the electrode surface, respectively. Then, at $0.4 \mathrm{~V}$, the small semicircle in the intermediate frequency region disappears, and

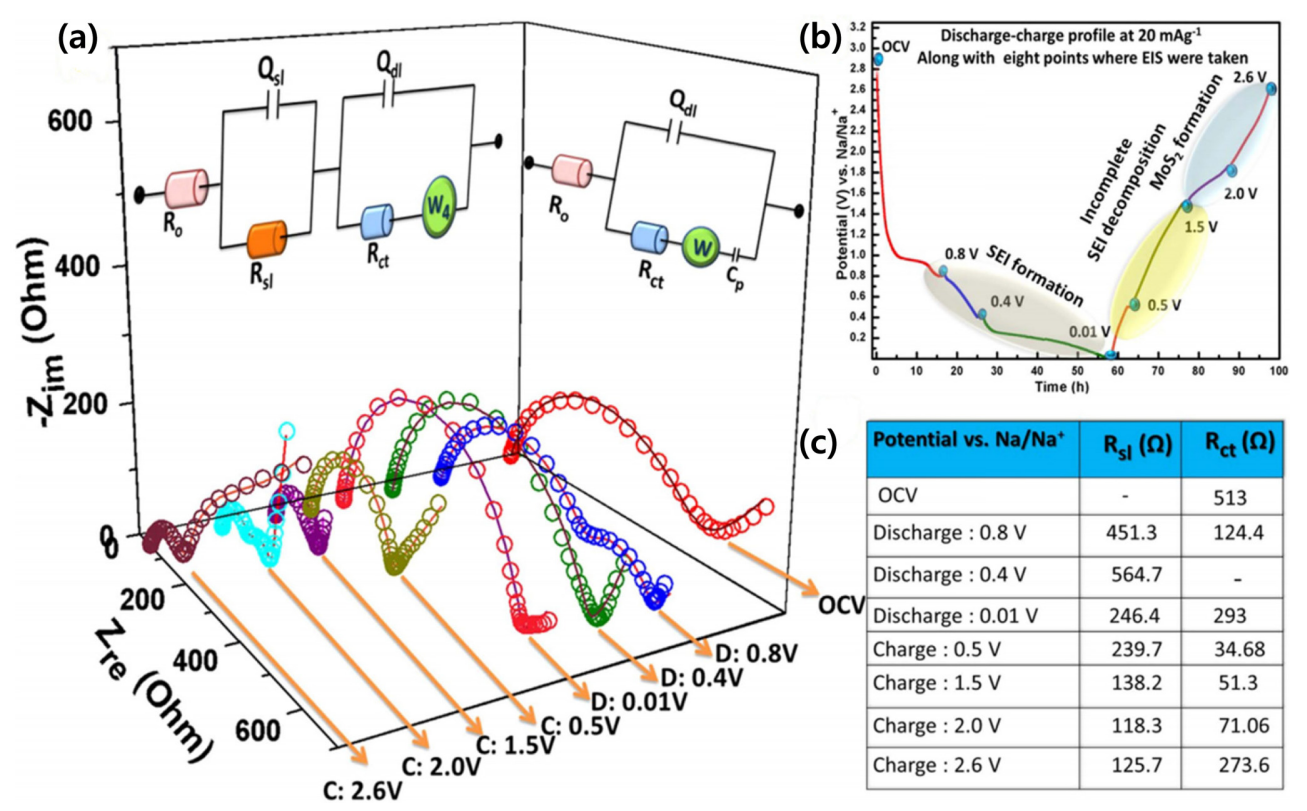

Fig. 10. (a) EIS (Nyquist plot) spectra of the $\mathrm{MoS}_{2}$ sodium conversion system at different states of charge/discharge. (b) The charge-discharge profile of the $1^{\text {st }}$ cycle with the point of the impedance measurement point. (c) $R_{s l}$ and $R_{c t}$ values from the equivalent circuit model fitting. Reprinted with permission from Ref. [50]. Copyright ( $) 2015$ Nature Publishing Group. 
a semicircle with a high $\mathrm{R}_{\mathrm{sl}}$ (migration resistance through SEI layer) value was observed due to the formation of a thick SEI layer on the surface and distorting of the host material. At the final stage of discharge, $0.01 \mathrm{~V}$, a single semicircle in the entire frequency range, dominates the impedance value of the SEI layer. However, during charging, $\mathrm{R}_{\mathrm{sl}}$ at $0.5-1.5 \mathrm{~V}$ starts to decrease dramatically and continues to decline until the end of the charging process. This phenomenon indicates that the thickly formed SEI layer was dissolved or destroyed during discharge due to the volume change of the electrode material. As the electrode is charged to $2.0 \mathrm{~V}$, the semicircle correlating to the SEI layer is further reduced, and incomplete $\mathrm{MoS}_{2}$ formation generates a middle-frequency semicircle. When charged to $2.6 \mathrm{~V}$, the highfrequency region representing the SEI layer is stably maintained without any change. This partial decomposition of the SEI layer resulted in the extra capacity over the theoretical value.

The $\mathrm{R}_{\mathrm{SEI}}$ indicates resistance from an interfacial layer that is generated by the decomposition of the electrolyte. Initially, the studies on this SEI layer focused on understanding the degradation of graphite electrodes by revealing the relationship between the resistance characteristics of the SEI layer and the cycling conditions. However, in recent studies on nanoscale electrode materials for high-performance lithium-ion batteries, the SEI layer began to be considered as another factor resulting in extra capacity through reversible reactions. The reversible SEI formation with extra capacity has not yet been clarified in detail. Therefore, analyzing the SEI layer using EIS is very useful for observing not only the resistance of the anode material but also extra capacity from reversible SEI layer formation, which is generally called a polymeric gel-like film.

\subsection{Characterization of $\mathbf{R}_{\mathrm{ct}}$ in EIS spectra}

The second semicircle in the EIS results in Fig. 8 is related to the $R_{c t}$. $R_{c t}$ is linked to the kinetics of an electrochemical reaction and is changed by the surface coating, phase transition, bandgap structure and particle size. Moreover, $\mathrm{R}_{\mathrm{ct}}$ shows a dramatic change in response to a temperature change. In particular, at low temperatures $\left(\leq 20^{\circ} \mathrm{C}\right)$, the total resistance of the lithium-ion cell is determined by $\mathrm{R}_{\mathrm{ct}}$. Therefore, monitoring $\mathrm{R}_{\mathrm{ct}}$ is useful for understanding electrode reaction processes that occur during the charge-dis- charge process and the characteristics of lithium-ion batteries depending on the temperature changes.

Lee et al. compared the $\mathrm{R}_{\mathrm{ct}}$ of pristine $\mathrm{LiNi}_{0.5+\mathrm{x}}$ $\mathrm{Co}_{0.2} \mathrm{Mn}_{0.3-\mathrm{x}} \mathrm{O}_{2}(\mathrm{x}=0,0.1$, and 0.2$)$ and clearly identified the impedance difference according to the Ni contents of the electrode material[51]. Each pristine electrode had only one charge transfer-related semicircle in the impedance spectra. The fitting results showed the lower Ni-content NCM (523) has a higher $\mathrm{R}_{\mathrm{ct}}$ value of $127.42 \Omega$, and the higher Ni-content NCM (721) has a lower $R_{c t}$ value of $79.45 \Omega$. This report suggested that the increase in the Ni contents in the Co-fixed NCM system will have a beneficial effect on the electron conductivity, showing $\mathrm{R}_{\mathrm{ct}}$ dependence on the transition metal composition. In addition, Zhang et al. applied the EIS analysis to confirm the $\mathrm{R}_{\mathrm{ct}}$ dependence on voltage and temperature [52]. The $\mathrm{Ni}$-based cathode/graphite cell was cycled in the voltage range of 3.1-4.2 V, and it was found that $R_{c t}$ changed more than $\mathrm{R}_{\mathrm{b}}$ or $\mathrm{R}_{\mathrm{SEI}}$. $\mathrm{R}_{\mathrm{ct}}$, which accounts for more than $60 \%$ of the total cell resistance, predominantly determines the cell resistance. Especially below $3.0 \mathrm{~V}$, the ratio of $\mathrm{R}_{\mathrm{ct}}$ to the total resistance exceeds $90 \%$ and reaches almost $100 \%$ in a completely discharged state. Ogihara et al. reported that the tendency of cell resistance changes at porous electrodes depends on the temperature by using four parameters: electric resistance $\left(\mathrm{R}_{\mathrm{e}}\right)$, electrolyte bulk resistance $\left(R_{\text {sol }}\right)$, ionic resistance in pores $\left(R_{\text {ion }}\right)$, and charge transfer resistance for lithium intercalation $\left(\mathrm{R}_{\mathrm{ct}}\right)$ in Fig. 11(a) [53]. The plots can be described as TLM for cylindrical pores for faradaic processes at all temperatures. Upon decreasing temperature, the real impedance component at $100 \mathrm{kHz}$, which determines the value of $\mathrm{R}_{\text {sol }}$, slightly shifts toward more positive values, and the length of the $45^{\circ}$ slope line, which reflects $R_{\text {ion, }}$ increases, as shown in Fig. 11(b). This indicates that two resistances somewhat increase with decreasing temperature. However, the semicircle in the low-frequency region, which corresponds to $R_{c t}$, is significantly enlarged with decreasing temperature. It seems that the temperature dependence of $\mathrm{R}_{\mathrm{ct}}$ is larger than that of $\mathrm{R}_{\text {sol }}$ or $\mathrm{R}_{\mathrm{ion}}$. Based on recorded EIS spectra, fitting was performed for a clear comparison of each resistance parameter. As shown in Fig. 11(c), $R_{e}$ is approximately $2 \sim 4$ orders smaller in magnitude than the others, and there is only a small change in temperature. $\mathrm{R}_{\text {ion }}$ and $\mathrm{R}_{\text {sol }}$ have relatively large values and moderate temperature 

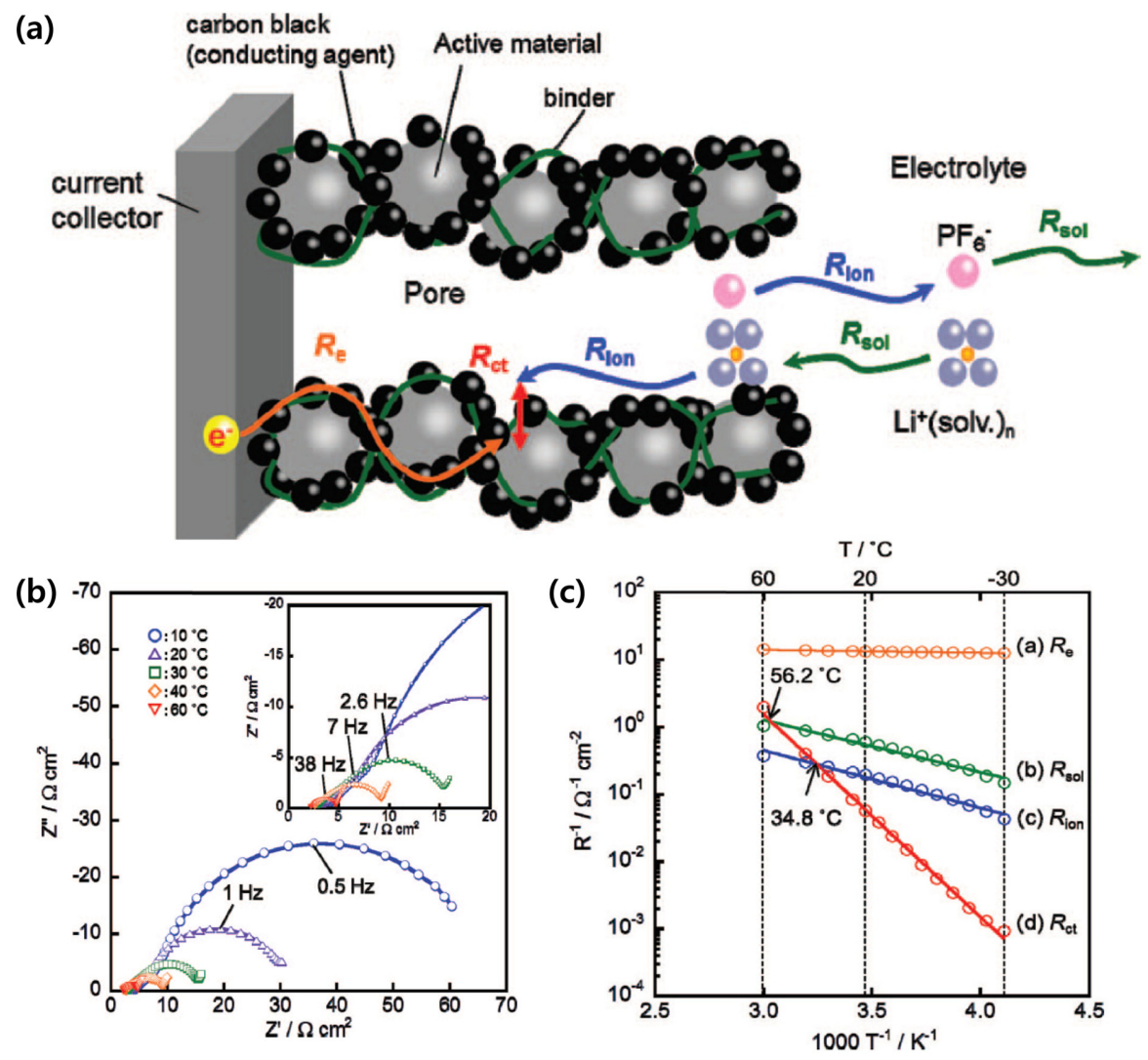

Fig. 11. (a) Schematic illustration of the respective resistances at porous electrodes. (b) Nyquist plots for symmetric cells using two identical electrodes at various temperatures. (c) Temperature dependence of each resistance obtained by the fitting. Reprinted with permission from Ref. [53]. Copyright (C) 2012 The electrochemical Society.

dependencies. On the other hand, $\mathrm{R}_{\mathrm{ct}}$ exhibits a strong temperature dependence and becomes the largest resistance value among the four resistance parameters in the electrode when the temperature is lower than $34.8^{\circ} \mathrm{C}$.

Ogihara et al. investigated the relationship between the energy density and power density of $\mathrm{LiNiO}_{2}$ based porous electrodes by monitoring the resistance change according to the coated electrode thickness on aluminum foil [54]. A symmetric cell was assembled to remove the effects of the $\mathrm{Li}$ counter electrode, and the TLM was used to separate $R_{\text {ion }}$ and $R_{\mathrm{ct}}$ from the porous electrode resistance. The electrochemical results for various thicknesses $(25 \sim 75 \mu \mathrm{m})$ show that as the thickness increases, the energy density increases while the power density decreases. The improved energy density is easily understood as an increase in the portion of the active material relative to the total cell volume. However, the cause of the power density change is divided into two regions according to decreasing tendency: region I (thin electrode), the power density slightly decreases; region II (thick electrode), the power density dramatically decreases. These two separate regions are determined by the correlation between $\mathrm{R}_{\mathrm{ct}}$ and $\mathrm{R}_{\mathrm{ion}}$, which accounts for the largest portion of the total cell resistance. The EIS fitting results demonstrate that the $\mathrm{R}_{\mathrm{ct}}$ value is the main influence on the thin electrode, while the $R_{\text {ion }}$ value is the dominant factor in the thick electrode. Since the $R_{c t}$ value is inversely proportional to the charge-transfer active area, it gradually decreases as the thickness increases. Conversely, because the $\mathrm{R}_{\text {ion }}$ value increases in proportion to the lithium diffusion length, the larger the thickness is, 
the larger the amount of $\mathrm{R}_{\text {ion }}$. Therefore, the power density slightly decreases in region I because only $R_{c t}$ affects the rate capability. However, region II shows a dramatic decrease in power density since an increase in $R_{c t}$ as well as $R_{\text {ion }}$ affects the power density.

$\mathrm{R}_{\mathrm{ct}}$ is the largest value among the total cell resistance at room temperature. The power density of a lithium-ion battery is mainly determined by $R_{c t}$. To reduce the $R_{c t}$ value, many studies have examined the effect of electrode properties such as the Ni content, temperature and electrode thickness on the $\mathrm{R}_{\mathrm{ct}}$ value. In addition, since $\mathrm{R}_{\mathrm{ct}}$ varies depending on the charge/ discharge state, it can contribute to understanding the reaction mechanism by comparing the resistance changes over voltage ranges. Therefore, an analysis of the $\mathrm{R}_{\mathrm{ct}}$ value would be helpful not only in understanding the reaction mechanism of the lithium-ion battery but also in research for the optimization of the cycling performance of commercial cell systems.

\subsection{Characterization of Warburg in EIS spectra}

The straight line immediately after the semicircles in Fig. 8 represents the Warburg impedance associated with the lithium-ion diffusion process of the electrode material. The $\mathrm{D}_{\mathrm{Li}+}$ calculation generally proceeds using the following equation proposed by Ho et al. [55].

$$
\begin{aligned}
& D_{L i+}=\frac{1}{2}\left[\left(\frac{V_{M}}{S F \sigma}\right) \times\left(\frac{\partial E}{\partial x}\right)\right]^{2} \\
& \cdot \mathrm{V}_{\mathrm{M}}: \text { mole volume of active material } \\
& \cdot \mathrm{F}: \text { Faraday constant }\left(96,486 \mathrm{C} \mathrm{mol}^{-1}\right) \\
& \cdot \mathrm{S}: \text { electrode/electrolyte contact area } \\
& \cdot \sigma: \text { the slope of } \mathrm{Z}_{\text {img }} \text { Vs. } 1 / \sqrt{2} \pi f \\
& \cdot \delta \mathrm{E} / \delta \mathrm{x} \text { : the slope of charge/discharge curves }
\end{aligned}
$$

Using equation (7), the diffusion coefficient can be easily calculated from the low-frequency area. However, since the above equation does not consider the phase transition of the electrode material, the calculation of the diffusion coefficient is only possible for solidsolution behavior (i.e., the single-phase region) [56]. Accordingly, the Warburg impedance analysis is mainly used to calculate the diffusion coefficient of a cathode material that shows a single-phase reaction, combined with galvanostatic intermittent titration (GITT), potentiostatic intermittent titration technique (PITT) and cyclic voltammetry (CV)[57-60]. Xia et al. measured the chemical diffusion coefficients of $\mathrm{c}$-axis oriented
$\mathrm{LiCoO}_{2}$ thin films using EIS and PITT and clearly observed the order/disorder transition phase boundaries near the composition $\mathrm{Li}_{0.5} \mathrm{CoO}_{2}$ [57]. Liu et al. synthesized a $\mathrm{LiFePO}_{4} / \mathrm{C}$ nanocomposite and confirmed the lithium intercalation and deintercalation diffusivity by $\mathrm{CV}$ and EIS [60]. Xie et al. prepared a $\mathrm{LiMn}_{2} \mathrm{O}_{4}$ thin film by pulse laser deposition on stainless-steel substrates and characterized the lithium diffusion using CV, PITT, and EIS [61]. Rui et al. measured the chemical diffusion coefficients of lithium ions $\left(\mathrm{D}_{\mathrm{Li}+}\right)$ in $\mathrm{Li}_{3} \mathrm{~V}_{2}\left(\mathrm{PO}_{4}\right)_{3}$ by dividing the system into a two-phase region and single-phase region [62]. The chemical diffusion coefficient of the two-phase region was measured through GITT and CV. The single-phase region from 4.70 to $3.68 \mathrm{~V}\left(\mathrm{Li}_{2.5} \mathrm{~V}_{2}\left(\mathrm{PO}_{4}\right)_{3} \rightarrow \mathrm{Li}_{3} \mathrm{~V}_{2}\left(\mathrm{PO}_{4}\right)_{3}\right)$ during the discharge process was calculated integrally using GITT, $\mathrm{CV}$, and EIS techniques. In addition, the Warburg impedance has been used to calculate the chemical diffusion coefficient for negative electrode materials such as Si. Ding et al. measured the diffusion coefficient by EIS as mentioned above and compared it to the results from different analysis techniques, such as $\mathrm{CV}$ and GITT methods[63]. The diffusion coefficients of lithium ions in nano-Si at different charge-discharge states were determined by EIS and GITT. The $\mathrm{D}_{\mathrm{Li}^{+}}$values calculated from EIS range from $10^{-13}$ to $10^{-12} \mathrm{~cm}^{2} \mathrm{~s}^{-1}$. The results from GITT are slightly higher than those from EIS due to its nonequilibrium states (30 minutes was given before measurement for stabilization in EIS, but GITT gave only 10 minutes). Although the actual values of the EIS and GITT analytical methods were different, it was found that the general trend in the graph shown by the variation in the lithium concentration in the silicon electrode was the same as a "W" type.

The Warburg impedance at low-frequency is directly related to the diffusion of lithium-ions. However, there are limitations to EIS measurements at two-phase transitions during lithium insertion/ extraction. Therefore, it is necessary to comprehensively use several techniques for accurate analysis.

\section{Conclusion and Outlook}

This mini-review outlines the basic principles of EIS analysis and how to construct a physically sound circuit model. In addition, it introduces the practical applications of the EIS technique. The contents of this review are summarized as follows. 
(1) $R_{b}$, the initial $x$-axis intercept value of the Nyquist plot, is the total resistance of the electrolyte, separator, and electrodes. This value is significantly related to the state of health.

(2) $\mathrm{R}_{\mathrm{SEI}}$, the first semicircle in the Nyquist plot, is associated with the generation of the interfacial layer deposited on the electrode. This value can be used to analyze the formation of the SEI layer, which is the result of the decomposition of the electrolyte. Additionally, the formation of polymeric gel-like films frequently shown in nano-sized electrode materials can be observed through EIS analysis.

(3) $R_{c t}$, the second semicircle in the Nyquist plot, is related to the kinetics of the electrochemical reaction, which is changed by the surface coating, phase transition, band gap structure or particle size. This value represents the faradic charge-transfer resistance and is used to clarify the reaction mechanism or temperaturedependent characteristics of lithium-ion batteries.

(4) Finally, the Warburg impedance is the final straight line of the Nyquist plot, which is related to the diffusion of lithium ions.

Numerous electrochemical analysis methods can be used to investigate the internal processes of a battery. However, in the case of the EIS analysis, it is possible to obtain important values from each component in a lithium-ion cell in a single experiment. Moreover, it is a nondestructive method that does not damage the battery. Additionally, it takes a relatively short time to carry out the measurement. If a precise circuit model is established and a uniform cell system is constructed to perform an EIS analysis, crucial information about each component in the lithium-ion battery will be obtained. Because measurements can be easily carried out regardless of battery cell conditions and sizes, EIS is an efficient analysis tool capable of investigating the reaction and degradation mechanisms of battery systems.

\section{Acknowledgment}

This work was supported by the National Research Foundation of Korea (NRF) grant funded by the Korea government (MSIP) (No. NRF-2017R1A4A1015770). This work was supported by the National Research Foundation of Korea (NRF) grant funded by the Korea government (MSIP) (No. NRF-2019R1A2C2003731). W.C. acknowledges NRF (National Research Foundation of Korea) Grant funded by Korean Government
(NRF-2016H1A2A1910157-Global Ph.D. Fellowship Program).

\section{Supporting Information}

Supporting Information is available at https://doi.org/ $10.33961 /$ jecst.2019.00528

\section{References}

[1] O. Heaviside, The Electriian., reprinted as Electrical Papers, 1886, 212.

[2] E. Warburg, Ann. Phys. Chem., 1899, 3, 493.

[3] A.E. Thiessen, Gen. Radio Exp., 1933, 7, 7-9.

[4] D.C Grahame, Chem. Rev., 1947, 41(3), 441-501.

[5] V.D.D. MacDonald, Plenum Press. New York-London, 1977, 1, 358-359.

[6] D.E. Smith, H.H. Bauer, CRC Crit. Rev. Anal. Chem., 1971, 2(2), 247-343.

[7] C. Gabrielli, Tech. Rep. No 004, Solartron, Hampshire, $U K ., 1984,(3), 1-120$.

[8] M.J. Ross, K.R. William, Impedance Spectroscopy: Emphasizing Solid Materials and Systems; John Wiley \& Sons: John Wiley Sons New York, 1987.

[9] Y.H. Kim, Y.S. Kwon, M.Y. Shon, M.J. Moon, J. Electrochem. Sci. Technol., 2018, 9(1), 1-8.

[10] N.N. Hazani, Y. Mohd, S. Ahmad, I. Sheikh, M. Ghazali, Y. Farina, N.N. Dzulkifli, J. Electrochem. Sci. Technol., 2019, 10(1), 29-36.

[11] H.-B. Choe, H.-S. Lee, M.A. Ismail, M.W. Hussin, Int J Electrochem Sci., 2015, 10, 9775-9789.

[12] K. Pandey, S.T.A. Islam, T. Happe, F.A. Armstrong, $F$. A. Proc. Natl. Acad. Sci. U. S. A, 2017, 114(15), 38433848.

[13] A. Papaderakis, D. Tsiplakides, S. Balomenou, S. Sotiropoulos, J. Electroanal. Chem., 2015, 757, 216-224.

[14] A.S. Dezfuli, M.R. Ganjali, H.R. Naderi, P. Norouzi, RSC Adv., 2015, 5, 46050-46058.

[15] W. Du, Z. Wang, Z. Zhu, S. Hu, X. Zhu, Y. Shi, H. Pang, X. Qian, J. Mater. Chem. A, 2014, 2, 9613.

[16] E.K. Park, J.W. Yun, J. Electrochem. Sci. Technol., 2016, 7(1), 33-40.

[17] A. Bertei, E. Ruiz-trejo, F. Tariq, V. Yufit, A. Atkinson, N.P. Brandon, Int. J. Hydrogen Energy, 2016, 41, 2238122393.

[18] X. Zhang, W. Wu, Z. Zhao, B. Tu, D. Ou, D. Cui, M. Cheng, Catal. Sci. Technol., 2016, 6, 4945-4952.

[19] X. Hu, S. Li, H. Peng, J. Power Sources, 2012, 198, 359-367.

[20] T. Momma, M. Matsunaga, D. Mukoyama, T. Osaka, $J$. Power Sources, 2012, 216, 304-307.

[21] A. Barai, K. Uddin, W.D. Widanage, A. McGordon, P. Jennings, Sci. Rep., 2018, $8(21)$.

[22] U. Krewer, F. Röder, E. Harinath, R.D. Braatz, B. Bedürftig, R. Findeisen, J. Electrochem. Soc., 2018, 
165(16), A3656-A3673

[23] S. Rodrigues, N. Munichandraiah, A.K Shukla, $J$ Solid State Electrochem., 1999, 3, 397-405.

[24] S. Kochowski, K. Nitsch, Thin Solid Films, 2002, 415, 133-137.

[25] S.P. Jing, J.G. Love, S.P.S. Badwal, Key Eng. Mater, 1997, 125-126, 81-132.

[26] J.E.B. Randle, Discuss. Faraday Soc., 1947, 1, 11-19.

[27] M. Gaberscek, J. Moskon, B. Erjavec, R. Dominko, J. Jamnik, Electrochem. Solid-State Lett., 2008, 11(10), A170-A174.

[28] U. Westerhoff, K. Kurbach, F. Lienesch, M. Kurrat, Energy Technol., 2016, 4, 1620-1630.

[29] T. Momma, T. Yokoshima, H. Nara, Y. Gima, T. Osaka, Electrochim. Acta, 2014, 131, 195-201.

[30] O.S. Mendoza-Hernandez, H. Ishikawa, Y. Nishikawa, Y. Maruyama, Y. Sone, M. Umeda, Electrochim. Acta, 2014, 131, 168-173.

[31] D. Andre, M. Meiler, K. Steiner, H. Walz, T. Soczkaguth, D.U. Sauer, J. Power Sources, 2011, 196(12), 5349-5356.

[32] P. Gao, C. Zhang, G. Wen, J. Power Sources, 2015, 294, 67-74.

[33] B.T. Habte, F. Jiang, Solid State Ionics, 2018, 314, 8191.

[34] H. Nara, D. Mukoyama, R. Shimizu, T. Momma, T. Osaka, J. Power Sources, 2019, 409, 139-147.

[35] D.W. Abarbanel, K.J. Nelson, J.R. Dahn, J. Electrochem. Soc., 2016, 163(3), A522-A529.

[36] N.S. Zhai, M.W. Li, W.L. Wang, D.L Zhang, D.G. Xu, J. Phys. Conf. Ser, 2006, 48, 1157.

[37] M.D. Murbach, D.T. Schwartz, J. Electrochem. Soc., 2018, 165(2), A297-A304.

[38] A. Eddahech, O. Briat, J.-M Vinassa, J. Power Sources, 2014, 258, 218-227.

[39] S.F. Schuster, T. Bach, E. Fleder, J. Müller, M. Brand, G. Sextl, A. Jossen, J. Energy Storage, 2015, 1, 44-53.

[40] S.F. Schuster, M.J. Brand, C. Campestrini, M. Gleissenberger, A. Jossen, J. Power Sources, 2016, 305, 191-199.

[41] J. Vetter, P. Novák, M.R. Wagner, C. Veit, K.-C. Möller, J.O. Besenhard, M. Winter, M. Wohlfahrt-Mehrens, C. Vogler, A. Hammouche, J. Power Sources, 2005, 147, 269-281.

[42] K.M. Shaju, F. Jiao, A. lie Débart, P.G. Bruce, Phys. Chem. Chem. Phys., 2007, 9, 1837-1842.

[43] K.-A. Kwon, H.-S. Lim, Y.-K. Sun, K.-D. Suh, J. Phys.
Chem. C, 2014, 118, 2897-2907.

[44] S. Xu, C.M. Hessel, H. Ren, R. Yu, Q. Jin, M. Yang, H. Zhao, D. Wang, Energy Environ. Sci., 2014, 7, 632.

[45] D. Chen, H. Quan, J. Liang, L. Guo, Nanoscale, 2013, 5, 9684.

[46] Y. Xiao, X. Wang, W. Wang, D. Zhao, M. Cao, ACS Appl. Mater. Interfaces, 2014, 6, 2051-2058.

[47] S.S. Zhang, K. Xu, T.R. Jow, Electrochim. Acta, 2006, 51(8-9), 1636-1640.

[48] M. Steinhauer, S. Risse, N. Wagner, K.A. Friedrich, Electrochim. Acta., 2017, 228, 652-658.

[49] J.S. Gnanaraj, R.W. Thompson, S.N. Iaconatti, J.F. Dicarlo, K.M. Abraham, Electrochem. Solid-State Lett., 2005, 8(2), A128-A132.

[50] T.S. Sahu, S. Mitra, Sci. Rep., 2015, 5, 12571.

[51] W. Lee, S. Muhammad, T. Kim, H. Kim, E. Lee, M. Jeong, S. Son, J.-H. Ryou, W.-S. Yoon, Adv. Energy Mater, 2018, 8, 1701788.

[52] S.S. Zhang, K. Xu, T.R. Jow, Electrochim. Acta, 2004, 49(7), 1057-1061.

[53] N. Ogihara, S. Kawauchi, C. Okuda, Y. Itou, Y. Takeuchi, Y. Ukyo, J. Electrochem. Soc., 2012, 159(7), A1034-A1039.

[54] N. Ogihara, Y. Itou, T. Sasaki, Y. Takeuchi, J. Phys. Chem. C, 2015, 119(9), 4612-4619.

[55] C. Ho, I.D. Raistrick, R.A. Huggins, J. Electrochem. Soc., 1980, 127(2), 343-350.

[56] H. Xia, L, Lu, G. Ceder, J. Power Sources, 2006, 159, 1422-1427.

[57] X.H. Rui, N. Ding, J. Liu, C. Li, C.H. Chen, Electrochim. Acta, 2010, 55(7), 2384-2390.

[58] J. Xie, N. Imanishi, T. Matsumura, A. Hirano, Y. Takeda, O. Yamamoto, Solid State Ionics, 2008, 179(910), 362-370.

[59] S.B. Tang, M.O. Lai, L. Lu, J. Alloys Compd., 2008, 449(1-2), 300-303.

[60] H. Liu, C. Li, H.P. Zhang, L.J. Fu, Y.P. Wu, H.Q. Wu, J. Power Sources, 2006, 159(1), 717-720.

[61] J. Xie, T. Tanaka, N. Imanishi, T. Matsumura, A. Hirano, Y. Takeda, O. Yamamoto, J. Power Sources, 2008, 180(1), 576-581.

[62] X.H. Rui, N. Ding, J. Liu, C. Li, C.H. Chen, Electrochim. Acta, 2010, 55(7), 2384-2390.

[63] N. Ding, J. Xu, Y.X. Yao, G. Wegner, X. Fang, C.H. Chen, I. Lieberwirth, Solid State Ionics, 2009, 180(1-2), 222-225. 